\title{
Surveillance of SARS-CoV-2 Antibody of Patients in Local Affected Area During the Lockdown
}

\section{Yueting Tang}

Wuhan University Zhongnan Hospital

Jiayu Sun

Shantou University Medical College

yumeng yuan

Shantou University Medical College

Fen Yao

Shantou University Medical College

\section{Bokun zheng}

Wuhan University Zhongnan Hospital

\section{Gui Yang}

Wuhan University Zhongnan Hospital

\section{Wen Xie}

Wuhan University Zhongnan Hospital

\section{Guangming Ye}

Wuhan University Zhongnan Hospital

Zhen Li

Wuhan University Zhongnan Hospital

xiaoyang jiao ( $\nabla$ xyjiao@stu.edu.cn )

Shantou University Medical College https://orcid.org/0000-0002-7568-0595

Yirong Li

Wuhan University Zhongnan Hospital

Research article

Keywords: COVID-19, SARS-CoV-2, Sero-surveillance

Posted Date: February 19th, 2021

DOl: https://doi.org/10.21203/rs.3.rs-251832/v1

License: (c) (1) This work is licensed under a Creative Commons Attribution 4.0 International License. Read Full License 


\section{Abstract}

Background: Serosurvalence is crucial in estimating the range of SARS-CoV-2 infections, predicting the possibility of another wave, and decide on a vaccination strategy. To understand the herd immunity after the COVID-19 pandemic, the seroprevalence was measured in 3062 individuals with or without COVID-19.

Methods: The levels of SARS-CoV-2 antibody IgM and IgG were measured by the immuno-colloidal gold method.

Results: The mean seroprevalence for IgM and IgG in all participants was $2.81 \%$ and $7.51 \%$, respectively. The positive rate of IgG was significantly higher in women than in men $(P<0.05)$. The highest positive rate of IgM was observed in 41-60 years of age (3.49\%), while the highest seroprevalence for IgG was observed in persons $>60$ years of age (8.61\%). The positive rates of IgM and IgG in the convalescent patients were $31.82 \%$ and $77.27 \%$, respectively, which was significantly higher than individuals with suspected syndromes or individuals without any clinical signs $(P<0.01)$. Seroprevalence for IgG in medical staff was markedly higher than those in residents. The seroprevalence in patients with various comorbidity was no significant difference $(P>0.05)$.

Conclusions: The low positive rate of the SARS-CoV-2 IgM and NA test indicated that the SARS-CoV-2 outbreak is subsiding after three months, and the possibility of reintroduction of the virus from an unidentified natural reservoir is low. Seroprevalence provides the information for humoral immunity and vaccine in the future.

\section{Introduction:}

COVID-19, caused by severe acute respiratory syndrome coronavirus-2 (SARS-CoV-2), has been inducing the global pandemic. The high morbidity and mortality have made it a significant threat to global health. Evidence suggested that SARS-CoV-2 can be transmitted effectively among humans, primarily by asymptomatic carriers through droplets or direct contact (1-4). The trend of an increasing rate mostly follows exponential growth. The mean primary reproduction number ( R0 ) was estimated to range from 2.24 to 3.58 , associated with two- to eight-fold increases in the reporting rate, and the epidemic was doubling time is 6.4 days $(95 \% \mathrm{Crl} 5.8-7.1$ days $)(5,6)$.

Due to no effective therapy, interrupt the transmission route by finding and isolating patients is an effective measure to control the disease. In the early stage of COVID-19, patients might manifest only transient febrile illness and minimal respiratory illness or be completely free of any clinical symptoms or signs. An unknown reservoir of the virus may exist in the sub-clinical individuals who may become a potential infection source, making it much more difficult to control the disease. Surveillance for infection was usually applied to address the transmission patterns, to observe latent infections, and to analyze disease progression. After strenuous efforts on epidemic control, the newly diagnostic cases have significantly reduced. However, in some areas, and the second outbreak has been more severe. 
Serosurvalence is crucial in estimating the range of SARS-CoV-2 infections, predicting the possibility of another wave, and decide on a vaccination strategy.

Neutralizing antibodies (NAbs) are critical components in the protective immune responses to viral infections because they can bind to viral particles and block them from entering the host cells $(7,8)$. NAbs are essential for protecting populations from re-infection. Information on the NAbs could be used to understand the epidemiology of SARS-CoV-2 infection and help determine the level of humoral immunity in patients. To COVID-19, different populations in different regions may have different humoral immunity. Wuhan was the epicenter of the COVID-19 of China, with the highest infectious rates; residents who lived in this city should have a high-risk for virus exposure. However, few data report residents' infection with certainty. As a tertiary university medical center in metropolitan Wuhan, Zhongnan hospital has a 3300bed capacity, and it serves about 100,000 people. Zhongnan hospital was designated a hospital responsible for COVID-19 patients' treatment during the pandemic. Many severe and critically severe patients were transferred there for intense therapy. Then, we selected this hospital to study the prevalence of COVID-19 infection. Given the relatively extraordinary exposure history of the individuals, including patients and hospital staff of this hospital, their seroprevalence may provide valuable information about the population infectious and their immune status. Seroprevalence of residents is vital for understanding the infectious population scale and their immune status and preventing disease spread and reemergence.

\section{Methods:}

Sample collection.

The study received ethics approval from the Ethics Committee of Zhongnan Hospital, Wuhan University. The blood samples were collected from a total of 3062 outpatients, including 2597 ordinary patients for COVID-19 screening, 355 individuals with suspected clinical symptoms, and 110 confirmed COVID-19 patients whose diagnosis was defined based on the New Coronavirus Pneumonia Prevention and Control Program (7th edition) published by the National Health Commission of China. Blood samples were obtained in containers without anticoagulant and kept at room temperature for $0.5 \mathrm{~h}$ to ensure serum separation. Serum samples were collected after being centrifuged at 4,000 rpm for $5 \mathrm{~min}$. Whether the participants had been exposed or the time of their exposure cannot be known with certainty. Therefore, a single blood sample was taken for antibody testing. Blood samples were collected after March 21, 2020, approximately two months after the outbreaks were recognized.

\section{Antibody and nucleic acid of SARS-CoV-2 Detection}

Serum IgM and IgG of SARS-CoV-2 was measured by immuno-colloidal gold technology (INNOVITA Biotechnology Company, Tangshan, China). 10uL serum samples diluted with two drops of sample diluent and then added in sampling well. Read the result within $15 \mathrm{~min}$. Both the control line and the test line appear simultaneously, indicating a positive outcome. Only show the control line, the result is negative, and if the control line does not appear, the result is invalid. At the same time, the nucleic acid 
(NA) of SARS-CoV-2 was measured by reverse transcription-polymerase chain reaction (RT-PCR) (DAAN GENE Company, Guangzhou, China). Also, all participants underwent a CT scan to confirm whether there were pathogenic lesions in the lung.

Statistical analyses.

Data were expressed as numbers and proportions. Differences in frequencies or proportions were tested using a $\chi 2$ test. SPSS 20.0 was used for statistical analyses (SPSS Incorporated, Chicago, IL, USA). P< 0.05 were statistically significant.

\section{Results:}

In total, 3062 individuals were enrolled in this study, of whom 1652 were males, and 1410 were females. Of 3062 samples, seroprevalence for $\operatorname{lgM}$ and $\lg G$ were $86(2.81 \%)$ and $230(7.51 \%)$, respectively. In persons with seropositive, 97 persons had positive CT scan results $(3.16 \%)$; only four persons $(0.13 \%)$ had NA positive, including two convalescent COVID-19 patients recovered in the observation period and two asymptomatic infection cases. The rate of specific antibody IgG was significantly higher in women than in men $(P<0.05)$.

Seroprevalence for IgM was significantly different among age group, from high to low: 41-60 years of age $(44[3.49 \%]),>60$ years of age $(23[3.36 \%]),<20$ years of age $(2[3.57 \%])$, and $21-40$ years of age (17 $[1.61 \%])$, respectively $(P<0.05)$. Seroprevalence for IgG among persons $>60$ years of age were $59(8.61 \%)$, 41-60 years of age were $104(8.24 \%), 21-40$ years of age were $65(6.14 \%)$, and persons $<=20$ years of age were $2(3.57 \%)$, respectively $(P=0.098)$.

We then grouped all subjects by their consulting department (Table 1), including Fever Clinic for patients (an outpatient clinic especially for the treatment of febrile or suspected patients), Fever Clinic for medical staff (clinic especially for screening highly suspected medical staff or the treatment of confirmed cases), Internal Medicine, Surgery department, Obstetrics/Gynecology/Fertility Clinic, Pediatric department, Oncology department, and another department. The highest seroprevalence for IgM observed in the Fever clinic, from high to low: Fever clinic for patients(10.34\%), Internal medicine (1.51\%), Surgery department (1.37\%), Oncology department (1.15\%), General department or other (1.01\%), Obstetrics/Gynecology/Fertility department (0.74\%), Fever clinic for medical staffs ( $0 \%)$, and Pediatric department $(0 \%)$, respectively $(P<0.001)$. IgG positive rate (from high to low): Fever clinic for medical staffs (25.0\%), Fever clinic (23.73\%), General department or other (8.08\%), internal medicine $(5.02 \%)$, Oncology department (3.07\%), Obstetrics/Gynecology/Fertility department (2.96\%), and Surgery department $(2.73 \%)(P<0.001)$ (Table 1$)$. 
Table 1

Positive Rate of SARS-CoV2 Among Different roups.

\section{$\mathrm{N}(\%)$}

\begin{tabular}{|c|c|c|c|c|c|}
\hline Characteristics & No.P & $\lg M(+)$ & $\lg G(+)$ & $\mathrm{NA}(+)$ & $\mathrm{CT}(+)$ \\
\hline \multicolumn{6}{|l|}{ Sex } \\
\hline Male & 1652 & $41(2.48)$ & $104(6.30)$ & $1(0.06)$ & $34(2.06)$ \\
\hline Female & 1410 & $45(3.19)$ & $126(8.94)$ & $3(0.21)$ & $63(4.47)$ \\
\hline$P$ & & 0.141 & 0.004 & 0.256 & 0.000 \\
\hline \multicolumn{6}{|l|}{ Age } \\
\hline$<=20$ & 56 & $2(3.57)$ & $2(3.57)$ & 0 & 0 \\
\hline $21-40$ & 1059 & $17(1.61)$ & $65(6.14)$ & 0 & $15(1.42)$ \\
\hline $41-60$ & 1262 & $44(3.49)$ & $104(8.24)$ & $3(0.24)$ & $49(3.88)$ \\
\hline$>60$ & 685 & $23(3.36)$ & $59(8.61)$ & $1(0.15)$ & $33(4.82)$ \\
\hline$P$ & & 0.035 & 0.098 & 0.461 & 0.000 \\
\hline \multicolumn{6}{|l|}{ Consulting room } \\
\hline Fever clinic for patient & 493 & $51(10.34)$ & $117(23.73)$ & $1(0.20)$ & $64(12.98)$ \\
\hline Fever clinic for medical staff & 16 & 0 & $4(25.00)$ & $1(6.25)$ & $3(18.75)$ \\
\hline Internal Medicine & 1395 & $21(1.51)$ & $70(5.02)$ & $1(0.07)$ & $23(1.65)$ \\
\hline Surgery department & 659 & $9(1.37)$ & $18(2.73)$ & $1(0.15)$ & $2(0.30)$ \\
\hline Obstetrics/Gynecology/Fertility & 135 & $1(0.74)$ & $4(2.96)$ & 0 & $2(1.48)$ \\
\hline Pediatric department & 4 & 0 & $1(25)$ & 0 & 0 \\
\hline oncology department & 261 & $3(1.15)$ & $8(3.07)$ & 0 & $2(0.77)$ \\
\hline General department/Other & 99 & $1(1.01)$ & $8(8.08)$ & 0 & $1(1.01)$ \\
\hline$P$ & & 0.000 & 0.000 & 0.000 & 0.000 \\
\hline \multicolumn{6}{|l|}{ Patient classification } \\
\hline COVID-19 convalescent & 110 & $35(31.82)$ & $85(77.27)$ & $2(1.82)$ & $51(46.36)$ \\
\hline SSC & 355 & $12(3.38)$ & $28(7.89)$ & 0 & $14(3.94)$ \\
\hline SAC & 1295 & $22(1.70)$ & $74(5.71)$ & $1(0.08)$ & $19(1.47)$ \\
\hline Attendance for other diseases & 1302 & $17(1.30)$ & $43(3.30)$ & $1(0.08)$ & $13(1.00)$ \\
\hline
\end{tabular}




\begin{tabular}{|c|c|c|c|c|c|}
\hline \multicolumn{6}{|c|}{$\mathbf{N}(\%)$} \\
\hline $\mathrm{P}$ & & 0.000 & 0.000 & 0.000 & 0.000 \\
\hline \multicolumn{6}{|l|}{ Comorbidity } \\
\hline Tumor & 387 & $7(1.81)$ & $14(3.62)$ & 0 & $5(1.29)$ \\
\hline CCD & 146 & $2(1.37)$ & $5(3.42)$ & 0 & $2(1.37)$ \\
\hline Digestive diseases & 259 & $2(0.77)$ & $11(4.25)$ & 0 & $2(0.77)$ \\
\hline Urogenital diseases & 141 & 0 & $3(2.13)$ & 0 & $1(0.71)$ \\
\hline Nervous diseases & 51 & $2(3.92)$ & $2(3.92)$ & $1(1.96)$ & $1(1.96)$ \\
\hline haematological diseases & 24 & $1(4.17)$ & $1(4.17)$ & 0 & 0 \\
\hline Other respiratory diseases & 64 & $1(1.56)$ & 0 & 0 & $1(1.56)$ \\
\hline External injury & 90 & $1(1.11)$ & $2(2.22)$ & 0 & 0 \\
\hline Pregnancy check-up & 68 & $1(1.47)$ & $2(2.94)$ & 0 & $1(1.47)$ \\
\hline AIDS & 13 & 0 & 0 & 0 & 0 \\
\hline Other & 59 & 0 & $3(5.08)$ & 0 & 0 \\
\hline$P$ & & 0.584 & 0.881 & 0.006 & 0.967 \\
\hline \multicolumn{6}{|c|}{$\begin{array}{l}\text { SSC: Screening for symptomatic condition (the most common symptoms related to COVID-19 } \\
\text { including fever, cough, chest tightness, diarrhea) }\end{array}$} \\
\hline \multicolumn{6}{|c|}{$\begin{array}{l}\text { SAC: Screening for asymptomatic condition (health examination professionals were asymptomatic } \\
\text { currently, but didn't rule out close contacts or had a symptom related to COVID-19) }\end{array}$} \\
\hline \multicolumn{6}{|c|}{ CCD: Cardiovascular and Cerebrovascular diseases } \\
\hline \multicolumn{6}{|c|}{ Other: including autoimmune diseases, skin diseases, stomatitis, laryngeal eyewinker, poisoning, etc } \\
\hline
\end{tabular}

The criteria for discharging patients, i.e., being afebrile for 14 days, clinical improvement, and the NA tested negative. In this study, we also included 110 patients discharged from the hospital to evaluate their immunity. The seroprevalence for IgM and IgG in the patients was 35 (31.82\%) and 85 (77.27\%), respectively, which was significantly higher as compared with those in other groups (Table 1). Among the 110 COVID-19 patients, most of the patients were convalescent, and their positive rate of IgG was marked higher than that of $\operatorname{lgM}$. Fourteen patients had IgM and IgG negative, while 11 cases were $\lg M$ positive, but IgG negative, maybe the newly infectious patients. CT scan showed that $46.36 \%$ of patients still have inflammatory lesions, indicating that it takes some time to absorb pulmonary inflammation. Forty-one patients with positive antibody detected the NAbs the second time for dynamic observation. Among them, 16 patients with IgM positive changed to negative on an average of 5 days, while six patients with IgG negative changed to positive in an average of 4.3 days. Three hundred fifty-five individuals with 
suspected syndromes, including fever, cough, chest congestion, and headache of undetermined origin, were included. The positive rate of IgM and IgG of them were 12 (3.38\%) and 28 (7.89\%), respectively. Their NA tests were negative, but 14 cases (3.94\%) had lung abnormality by CT. 1295 participants were currently asymptomatic. Still, some of them had recalled they might have a suspected symptom, without particular diagnosis due to mild symptoms, and chose to stay-at-home quarantine. Their seroprevalence for IgM and IgG were $22(1.71 \%)$ and 74 (5.74\%), respectively. Only one showed NA tests positive, and 19 (1.47\%) cases had lung abnormality by CT (Table 1$)$.

When we re-classify the participants according to their comorbidity, in 13 cases of AIDS patients, none of them had positive results in IgM, IgG, NA, and CT tests (Table 1). Three hundred eighty-seven tumor patients had IgM and IgG positive rate $7(1.81 \%)$ and 14 (3.62\%), respectively. One hundred forty-six patients with cardiovascular and cerebrovascular diseases, hypertension, and diabetes (CCD) had IgM and IgG positive rate $2(1.37 \%)$ and $5(3.42 \%)$, respectively. Two hundred fifty-nine patients with digestive system diseases had high levels of IgG 11 (4.25) but low levels of IgM (0.77). One hundred forty-one patients with urogenital system diseases had levels of $\operatorname{lgG} 3(2.13 \%)$, but none of IgM. Fifty-one patients with nerve system disease, including coma, syncope, disturbance of consciousness, dizziness, headache, epilepsy, spasm, neuralgia, and Alzheimer's disease, etc., had IgM and IgG positive rate $2(3.92 \%)$ and 2 (3.92\%), respectively. Twenty-four patients with hematological disease had IgM and IgG positive rate, were $1(4.17 \%)$, and $1(4.17 \%)$, respectively. Sixty-four patients with other respiratory disorders, including COPD, asthma, rhinitis, pharyngitis, bronchitis, and other pathogens lung infection, had IgM and IgG positive rate, were only $1(1.56 \%)$, and $0(0.00 \%)$, respectively. Ninety patients with external injury had IgM and IgG positive rate, were $1(1.11 \%)$, and $2(2.22 \%)$, respectively. Sixty-eight patients for pregnancy checkup had IgM and IgG positive rate, were 1 (1.47\%) and 2 (2.94\%), respectively. At last, another disease including autoimmune diseases, skin diseases, stomatitis, laryngeal eyewinker, poisoning, etc., had IgM and IgG positive rate, $0(0.00 \%)$ and $3(5.08 \%)$, respectively (Table 1$)$.

The variation of IgM and IgG in COVID-19 patients.

The percentage of patients who were IgM positive reduced after March 22, keeping at a low level after that. Seroprevalence for IgG peaked at $25.93 \%$ on March 23 due to many COVID-19 patients tested the NAbs on those days. As time passed, the positive rates of IgG remained at a low level; the range was from 2.82-14.08\% (Fig. 1A). Most patients were convalescent, and the positive rate of IgG was greater than that of IgM (Fig. 1A). As for subgroups, the positive rate of IgM had a higher level in the COVID-19 convalescent group (mean \pm SE: $33.41 \pm 0.34$ ) than SSC (mean \pm SE: $4.41 \pm 0.09$ ) and SAC groups (mean \pm SE: $1.28 \pm 0.09$ ). During all study periods, the COVID-19 convalescent group showed a higher IgG positive rate (mean \pm SE: $81.19 \pm 0.26$ ) than SSC (mean \pm SE: $7.35 \pm 0.05$ ) and SAC groups (mean \pm SE: $4.62 \pm 0.09)$ (Fig. 1B).

\section{Discussion:}


Due to its extreme high contagious, COVID-19 elicited a significant threat to global public health. It is of utmost importance to know whether the SARS-CoV-2 outbreak is subsiding after tremendous efforts on interrupting the human- to- human transmission to reduce secondary infections among close contacts and to prevent transmission amplification events. Currently, the first epidemic of COVID-19 is under control in Wuhan. Much work followed should be on preventing asymptomatic transmission. Some asymptomatic individuals might have existed; a new reservoir of the virus may exist that signals the need for continued surveillance. To investigate the humoral responses to the virus in the context of epidemiologic settings in the epicenter of COVID-19, we randomly selected individuals with or without suspected syndromes and convalescent COVID-19 patients from different consulting departments. Our results revealed a low positive rate of NA tests in the studied cohort. Seroprevalence for IgM and IgG in 355 individuals with suspected syndromes were significantly higher than those of 1295 asymptomatic participants (IgM and IgG were $3.38 \%$ vs. $1.70 \%$ and $7.89 \%$ vs. $5.17 \%$, respectively), indicating that viral infection in some cases only has mild or even no clinical manifestations. IgM is considered a parameter of the early phase of infection. IgM against SARS-CoV can be detected as early as in the first week(9). In our study, seroprevalence for IgM in ordinary patients for COVID-19 screening was more sensitive than the result of NA tests that was almost negative in our participants; the reason may be due to short phases of the virus shedding, or insufficient sample quality decrease the chances of detecting nucleic acids (10). IgM detection assays are incredibly valuable because it helps to find patients in the acute phase and to elucidate the range of subclinical infectious individual. In our study, the positive rate of IgM continues to decrease over time, consistent with a gradual decline in newly diagnostic cases. Combing to seroprevalence for IgM and positive rate of NA, the SARS-CoV-2 outbreak is subsiding, and the possibility of reintroduction of the virus from an unidentified natural reservoir will low.

The SARS-CoV-2 is transmitted by direct contact, droplets, feces, aerosols, or contaminated environmental surfaces. Therefore, the higher risk faced by a resident in the epicenter may be related in part to the temporal excretion pattern of the virus from COVID-19 patients. As we write this article, there is few data elucidate the infectious scale. Seroprevalence for IgG remained at the range was less than $10 \%$ in Wuhan residents, which is much lower than we expected. The finding of such low seroprevalence may be attributed to strictly precautionary measures, including home quarantine, temperature measurement, and must wear a surgical mask before entering the public place, washing hands frequently, etc. The highest seroprevalence for IgG was observed in COVID-19 convalescents. However, the positive rate of IgG was only $77.27 \%$ in convalescent patients. Eleven patients had IgG negative but IgM positive, suggesting they were newly infectious patients. Our data consistent with the previous reports, the presence of antibodies was $<40 \%$ among patients within 1-week since onset and rapidly increased to $94.3 \%$ (IgM) and $79.8 \%$ (IgG) since day-15 after onset (11).

In sixteen medical staffs who had suspected syndromes, the serological tests for IgM were entirely negative, the positive rate of IgG was $25 \%$, and the positive rate of NA was $6.25 \%$, indicating that latent infection had existed in these medical staffs. Seroprevalence for IgG in medical staff was significantly higher than those in residents. Before the outbreak was recognized, COVID-19 can be incredibly difficult to recognize due to the nonspecific nature of clinical manifestations, which may be the leading cause of 
high seropositive in medical staff. After the outbreak announced, all doctors entering isolation areas were required to follow the SOP about attire and to equip themselves with a double layer of personal protective equipment (PPE), including an N95 mask, covered with a full-face mask, goggles, a long-sleeve gown, a paper hat, and shoe covers. Our results revealed that personal protection was effective against viral infection, even in high-risk viral exposure.

NAbs are vital components in the protective immune responses to viral infections $(7,12)$. When evaluating the impact of comorbidity on immune responses, higher seroprevalence was observed in patients with tumors, cardiovascular and cerebrovascular diseases, hypertension, diabetes, nerve system disease, and digestive system diseases, but there was no significant difference among various groups. The seropositivity was higher in advanced age participants, the low prevalence in the younger populations. Higher seroprevalence of IgG was in the female than in the male, which may be because a man had low adaptive immune responses than a woman(13). A significant finding of our study is that none of the AIDS patients were infected. Either IgM or IgG was negative. Since there were only 13 patients, we could not rule out a sampling error. To COVID-19, information on immunity and pathogenesis is insufficient to provide a comprehensive basis for a specific drug or vaccine design. The observational data of NAbs may provide leads in controlling a possible reemergence of the disease.

Based on the transmission risk of known or unknown sources, infectious sources could not be ascertained. Patients without suspected syndromes were significantly more likely to be seronegative than those with the suspected syndrome. Moreover, participants who are come from the fever clinic had a significantly higher positive rate of IgM and IgG than other departments, demonstrating that the establishment of a fever clinic for suspected cases screening can improve the detection rate, achieve the goal of first isolation. The finding in asymptomatic seropositive persons indicates that the test will be useful in more extensive retrospective surveillance studies, which are needed to define the epidemiology and spectrum of disease fully (14).

\section{Conclusion:}

In the later period of the epidemic, the potential reintroduction of the virus from an unidentified natural reservoir remains a concern. Our results suggested that sero-surveillance is particularly valuable to trace hotspots of persons carrying antibodies to SARS-CoV and to track the origins of the disease. The low rate of SARS-CoV-2 antibodies in Wuhan residents indicated that the chain of human transmission could be successfully interrupted by public health measures.

\section{Abbreviations}




\begin{tabular}{|ll|}
\hline Acronym & Full title \\
\hline COVID-19 & Coronavirus disease 2019 \\
SARS-CoV-2 & Severe acute respiratory syndrome coronavirus-2 \\
RO & basic reproduction number \\
NAbs & Neutralizing antibodies \\
IgM & Immunoglobulin M \\
IgG & Immunoglobulin G \\
NA & nucleic acid \\
RT-PCR & reverse transcription-polymerase chain reaction \\
CCD & cardiovascular and cerebrovascular diseases, hypertension, and diabetes \\
COPD & chronic obstructive pulmonary diseases \\
SOP & Standard Operation Procedure \\
PPE & personal protective equipment \\
AIDS & Acquired Immune Deficiency Syndrome \\
\hline
\end{tabular}

\section{Declarations}

\section{Ethics approval and consent to participate}

This study was ethically approved in written form by the Ethics Committee of Central South Hospital. The written consents were obtained from study participants.

\section{Consent for publication}

Not applicable

\section{Availability of data and material}

Not applicable

\section{Competing interests.}

We declare no competing interests.

\section{Funding.}

This study was funded by the National Key Research and Development Program of China (grant number 2018YFE0204500), Zhongnan Hospital of Wuhan University Science, Technology and Innovation Seed 
Fund, Project znpy2019064, National Natural Science Foundation of China (Grant No. 81702273), Science and technology key project of Guangdong Province: Study on the source and epidemiology of COVID-19 (No.2020B111107001), Scientific research project of COVID-19 epidemic prevention and control in Guangdong universities (No.2020KZDZX1087), and Key Project for Anti-2019 Novel Coronavirus Pneumonia from the National Key Research and Development Program of China (Grant No. 2020YFC0845500).

\section{Author Contributions.}

TYT and XYJ designed and drafted the manuscript. YRL designed and revised the final manuscript. YRL ,TYT, and BKZ collected and summarized the clinical laboratory and clinical data. XW, YG, and LZ performed specimen collection antibody detection experiments. GMY is responsible for summarizing all data related to the virus. JYS ,YF , TYT and YMY processed statistical data. YRL had full access to all of the data in the study and take responsibility for the integrity of the data and the accuracy of the data analysis. YRL and XYJ contributed equally to this article and shared the corresponding authorship. All authors have read and approved the manuscript and ensure that this is the case.

\section{Acknowledgments}

We thank all of our colleagues on the front lines of diagnosing and treating COVID-19هWe thank all of the patients involved in the study.

\section{References}

1. Wang D, Hu B, Hu C, Zhu F, Liu X, Zhang J, et al. Clinical Characteristics of 138 Hospitalized Patients With 2019 Novel Coronavirus-Infected Pneumonia in Wuhan, China. JAMA. 2020.

2. Chang, Lin M, Wei L, Xie L, Zhu G, Dela Cruz CS, et al. Epidemiologic and Clinical Characteristics of Novel Coronavirus Infections Involving 13 Patients Outside Wuhan, China. JAMA. 2020.

3. Lai CC, Shih TP, Ko WC, Tang HJ, Hsueh PR. Severe acute respiratory syndrome coronavirus 2 (SARSCoV-2) and coronavirus disease-2019 (COVID-19): The epidemic and the challenges. Int J Antimicrob Agents. 2020;55(3):105924.

4. Biscayart C, Angeleri P, Lloveras S, Chaves T, Schlagenhauf P, Rodriguez-Morales AJ. The next big threat to global health? 2019 novel coronavirus (2019-nCoV): What advice can we give to travellers? Interim recommendations January 2020, from the Latin-American society for Travel Medicine (SLAMVI). Travel Med Infect Dis. 2020;33:101567.

5. Zhao S, Lin Q, Ran J, Musa SS, Yang G, Wang W, et al. Preliminary estimation of the basic reproduction number of novel coronavirus (2019-nCoV) in China, from 2019 to 2020: A data-driven analysis in the early phase of the outbreak. Int J Infect Dis. 2020;92:214-7.

6. Wu JT, Leung K, Leung GM. Nowcasting and forecasting the potential domestic and international spread of the 2019-nCoV outbreak originating in Wuhan, China: a modeling study. Lancet. 2020;395(10225):689-97. 
7. Daniel C, Anderson R, Buchmeier MJ, Fleming JO, Spaan WJ, Wege H, et al. Identification of an immunodominant linear neutralization domain on the $\mathrm{S} 2$ portion of the murine coronavirus spike glycoprotein and evidence that it forms part of complex tridimensional structure. J Virol. 1993;67(3):1185-94.

8. Crowe JE Jr, Suara RO, Brock S, Kallewaard N, House F, Weitkamp JH. Genetic and structural determinants of virus neutralizing antibodies. Immunol Res. 2001;23(2-3):135-45.

9. Yang Z, Wang S, Li Q, Li Y, Wei M, Gao H, et al. Determining SARS sub-clinical infection: a longitudinal seroepidemiological study in recovered SARS patients and controls after an outbreak in a general hospital. Scand J Infect Dis. 2009;41(6-7):507-10.

10. Meyer B, Drosten C, Muller MA. Serological assays for emerging coronaviruses: challenges and pitfalls. Virus Res. 2014;194:175-83.

11. Zeng Z, Chen L, Pan Y, Deng Q, Ye G, Li Y, et al. Re: Profile of specific antibodies to SARS-CoV-2: The first report. J Infect. 2020.

12. Kaplan M, Gawrieh S, Cotler SJ, Jensen DM. Neutralizing antibodies in hepatitis C virus infection: a review of immunological and clinical characteristics. Gastroenterology. 2003;125(2):597-604.

13. Gasmi A, Noor S, Tippairote T, Dadar M, Menzel A, Bjorklund G. Individual risk management strategy and potential therapeutic options for the COVID-19 pandemic. Clin Immunol. 2020:108409.

14. Hsueh PR, Kao CL, Lee CN, Chen LK, Ho MS, Sia C, et al. SARS antibody test for serosurveillance. Emerg Infect Dis. 2004;10(9):1558-62.

\section{Figures}


A

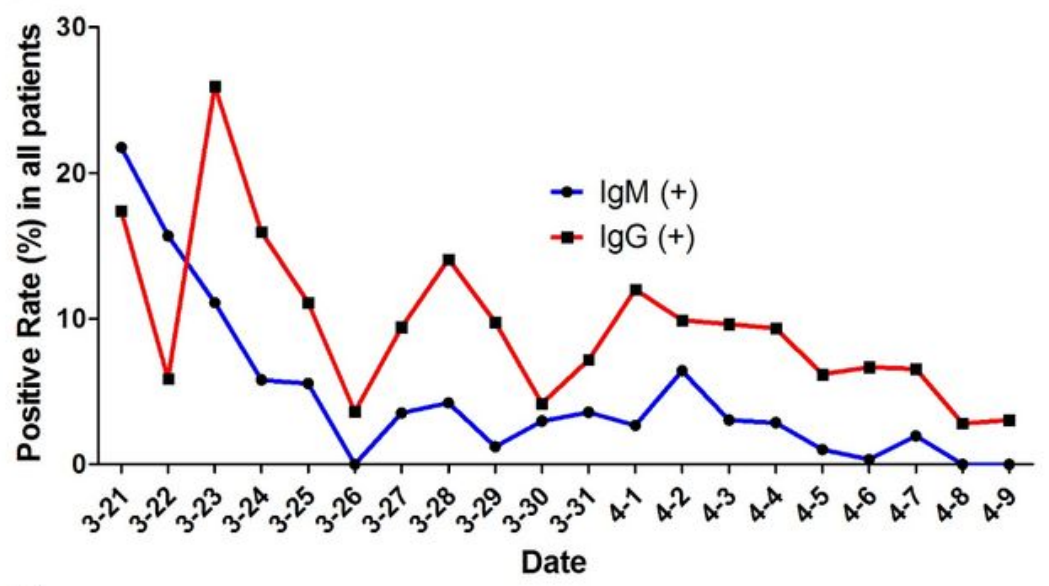

B

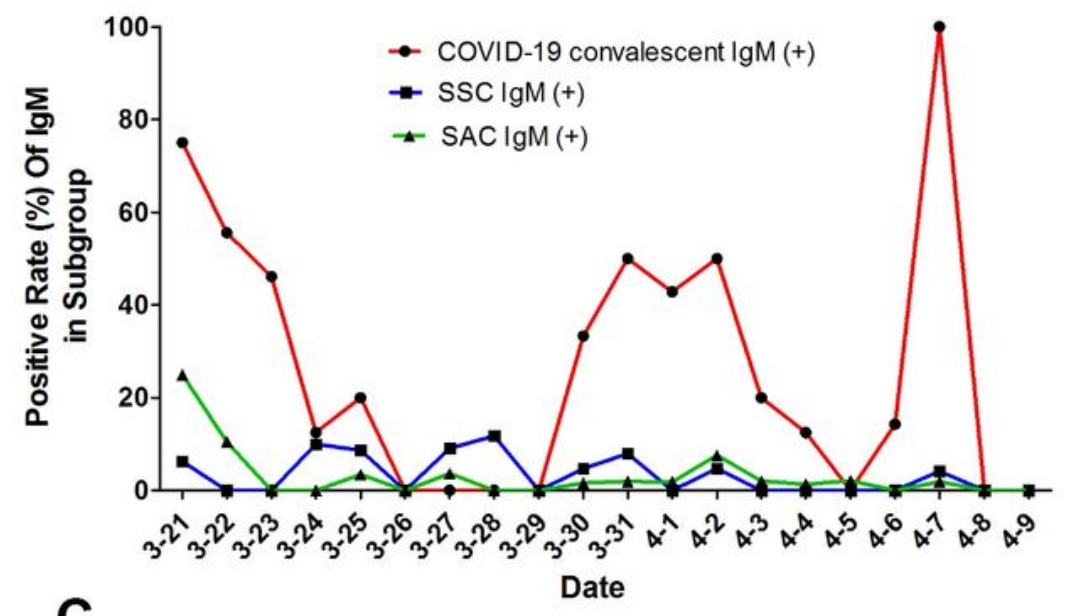

C

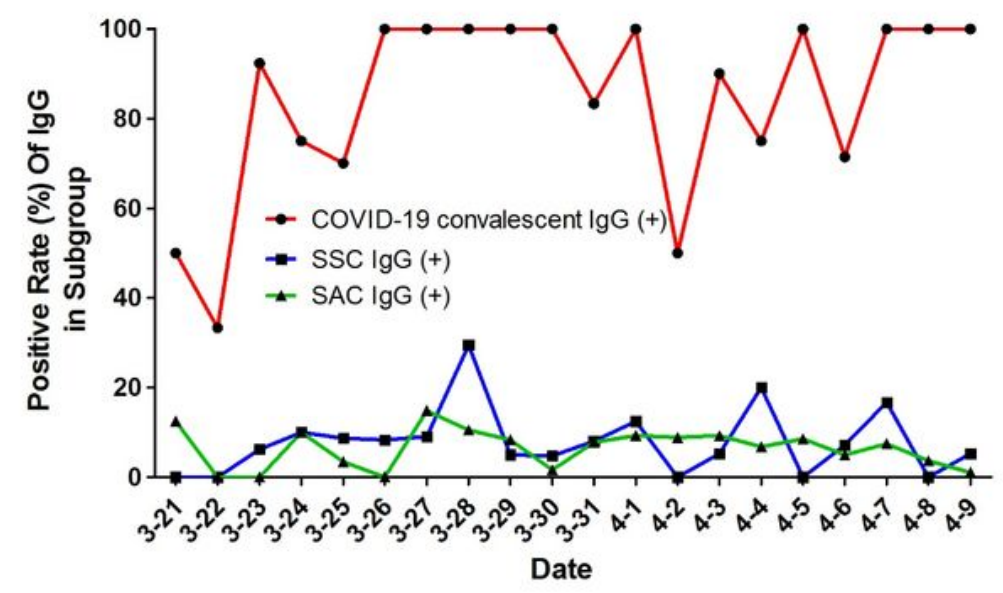

Figure 1

The temporal change of IgM and IgG in COVID-19 patients. (A) The curve showed that positive rates of $\operatorname{lgM}$ and IgG in patients that reduced after March 22. A small peak occurred on March 28 is due to the attendance of more COVID-19 convalescent and SAC patients at that time. (B) In general, a positive rate of IgM had a higher positive rate in COVID-19 convalescent than SAC and SCC groups. (C) During all study periods, the COVID-19 convalescent showed a higher positive IgG rate than SAC and SCC groups. 